\title{
Relative Dissociation Energy Measurements Using Ion Trap Collisional Activation
}

\author{
Kevin J. Hart and Scott A. McLuckey \\ Analytical Chemistry Division, Oak Ridge National Laboratory, Oak Ridge, Tennessee, USA
}

\begin{abstract}
The measured minimum resonance excitation amplitudes for decomposition of polyatomic ions in the quadrupole ion trap collisional activation experiment are shown to correlate with literature critical energies. The present article describes how experiments can be performed to derive threshold resonance excitation amplitudes via the kinetics associated with collision-induced dissociation (i.e., dissociation rate constants) in the quadrupole ion trap. The relationship between these threshold values and critical energies is established empirically by using kinetic data acquired for molecular ions with critical energies measured with other techniques. The experiments are complicated by the change in optimum resonance excitation frequency with amplitude, due presumably to contributions from higher order fields. It is proposed that the threshold resonance excitation amplitude is a measure of the change in temperature of the parent ion population required to achieve a measurable rate of decomposition. The present results indicate that the quadrupole ion trap may see new applications as a quantitative tool for the study of gaseous ion chemistry. (f Am Soc Mass Spectrom $1994,5,250-259$ )
\end{abstract}

$\mathrm{T}$ The minimum energy required for the fragmentation of a polyatomic ion is of fundamental importance in mass spectrometry. The so-called critical energy for the lowest energy decomposition pathway is an important factor, for example, in the observation, or lack thereof, of the molecular ion or pseudomolecular ion in the mass spectrum and the facility with which structural information can be obtained via tandem mass spectrometry (MS/MS). Apart from its effect on the behavior of an ion under various conditions in a mass spectrometer, the minimum critical energy is intimately related to the identity of the ion. It is therefore desirable to measure this quantity from both applied and fundamental points of view.

Critical energies associated with the decompositions of gaseous positive ions have been obtained primarily by threshold measurements involving ionization by electrons of well-defined energy or by photons $[1,2]$. Ihese experiments involve the measurement of the threshold for the appearance of a particular fragment ion from the parent molecule as a function of electron or photon energy. The critical energy is then taken as the difference between the measured appearance energy and the ionization potential of the molecule. These methods are generally restricted to the study of radical cations of species with relatively high volatilities, although results for radical anions can also be obtained in some cases. A much wider variety of ions can be studied via beam methods in which threshold mea-

Address reprint requests to Scott A. McLuckey, Oak Ridge National Laboratory, P.O. Box 2008, Oak Ridge, TN 37831-6365. surements can be acquired for mass-selected ions as a function of relative collision energy [3-5]. Beam methods generally involve some form of MS/MS in which ionization and ion activation are separate events. Therefore, any ion that can be formed in a mass spectrometer can be studied with beam methods. However, the parent ion population in a beam experiment is generally less well defined than the parent neutral population in experiments involving simultaneous ionization and excitation. The relative merits of the various approaches to making critical energy measurements are beyond the scope of this report. However, all techniques can suffer to varying degrees from potential errors due to kinetic shift [6], competitive shift [7], and thermal shift [8]. Kinetic shift arises from excess energy required to drive a reaction fast enough to be observed on the time frame of the measurement. Competitive shift arises from excess energy required to drive a reaction fast enough to compensate for the rate for some competitive reaction. The competitive shift is therefore of concern in measuring critical energies for reactions other than that of the lowest energy decomposition. Thermal shift arises from internal energy already present in the parent neutral or ion and tends to lead to an underestimate of the critical energy. In addition to these possibilities, the question of adiabatic versus vertical threshold values must also be considered in comparing and contrasting various approaches to critical energy measurements [1].

All of the techniques mentioned above involve activation of the neutral or ion in a time frame much shorter than that required for fragmentation. Relatively 
little work has been directed toward methods that involve a slow activation process relative to typical fragmentation rates. A notable exception is the work of Dunbar and Zaniewski $[9,10]$ in which low power continuous-wave laser irradiation of gaseous ions is being developed for evaluating dissociation thresholds. The collisional activation process in the quadrupole ion trap is, in many ways, very similar to the slow multiphoton irradiation experiment mentioned above as well as the sustained off-resonance irradiation technique described by Jacobson and coworkers [11]. These ion activation processes all occur via many discrete activation events with relatively long periods between events. All three processes also involve the possibility for cooling mechanisms other than fragmentation. In the rarefied environment of the ion cyclotron resonance spectrometer, infrared photon emission may be the most important cooling mechanism, whereas in the ion trap operated with roughly 1 mtorr of helium bath gas, both collisional and radiative cooling can occur. The slow multiphoton dissociation experiment [12], the ion trap collisional activation experiment [13], and the sustained off-resonance irradiation experiment have all been noted for their abilities to promote the lowest energy decomposition pathway. Indeed, the slow nature of activation in these experiments can make the loss of large fractions of the parent ion population via the lowest energy decomposition pathway difficult to avoid [14].

Recently, March and co-workers [15] have recognized that collision-induced dissociation as it is typically effected in the quadrupole ion trap can be characterized by the kinetics of parent ion decomposition under various experimental conditions. Such an approach may be useful, for example, in facilitating interlaboratory comparisons in MS/MS experiments. We were intrigued by this approach because of its potential for allowing for quantitative measurements of critical energies of decomposition for the wide variety of ions that can be stored in an ion trap. Such a capability would significantly enhance the value of the ion trap as a tool for fundamental research in gaseous ion chemistry and might also prove useful analytically in isomer differentiation. To investigate the possibility of measuring critical energies, or some parameter that can be related to critical energies, we have studied a number of parent ions with critical energies measured by other means. Parent ions are subjected to collisional activation by dipolar excitation at their fundamental secular frequencies [16] for various durations and as a function of amplitude of the excitation signal. A threshold resonance excitation amplitude is determined from these data. Comparison of these thresholds with literature critical energies indicates that the experimentally derived minimum resonance excitation amplitude is indeed related to kinetic stability of the ion. We describe here in detail the method for determining threshold resonance excitation amplitude along with our results with ions of known critical energy.
This work constitutes a first step in developing a means for quantifying ion stability with a quadrupole ion trap. Work continues on developing a model to understand the meaning of the threshold resonance excitation amplitude as it relates to critical energies derived by other means [17]. That is, we ask what roles might kinetic, competitive, and thermal shifts play in these experiments and how might we relate directly the value of the threshold excitation amplitude to critical energy without recourse to an empirical correlation.

These studies have also revealed other phenomena relevant to resonance excitation experiments in the ion trap including both ion activation and ion ejection. Shifts of parent ion secular frequencies, for example, can be significant under various collisional activation conditions [18]. We describe how these shifts are dealt with in determining collisional activation rates. Furthermore, the shape of the threshold resonance excitation versus literature critical energy curve reveals information on internal energy deposition as a function of resonance excitation amplitude. We also show that the measurement of threshold resonance excitation amplitude is independent of well depth but that the rate of internal energy deposition is highly dependent on well depth. The latter observatior has been made based on the appearance of ion trap tandem mass spectra [19] of other molecules and is now also shown to be reflected directly in the rate of energy deposition.

\section{Experimental}

\section{Samples}

Samples of allyl chloride [107-05-1], toluene [108-88-3], 1-methylnaphthalene [90-12-0], styrene [100-42-5], and ethylbenzene [100-41-4] were obtained from a Chem Services Kit (West Chester, PA) and were at least 99\% pure. Tetraethylsilane [631-36-7] (Aldrich Chemical Co., Milwaukee, WI), n-butylbenzene [104-51-8] (Eastman Kodak Co., Rochester, NY), and nitromethane [75-52-5] (Fischer Scientific Co., Fair Lawn, NJ) were at least 95\% pure. All of these samples were liquids. Sample vapors were introduced to the vacuum chamber of the instrument via a heated variable leak valve after two freeze/pump/thaw cycles. Sample pressures were measured by an ionization gauge (Granville-Phillips, Boulder, $\mathrm{CO}$, Model 280) and were maintained at a pressure of approximately $5 \times 10^{-7}$ torr (uncorrected). Background pressures in the instrument were typically $8 \times 10^{-8}$ torr (uncorrected).

\section{Instrumentation}

All experiments were performed on a Finnigan ion trap mass spectrometer (ITMS) (Finnigan MAT, San Jose, CA) with the vacuum chamber maintained at 100 ${ }^{\circ} \mathrm{C}$. The helium buffer gas was set at 1.14 mtorr using an ionization gauge and applying an ionization correc- 
tion factor of 0.14 [20]. The details of the operation of an ion trap mass spectrometer have been given elsewhere [21]. Molecular cations for each chemical system were formed by electron ionization. The basic scan function used for these experiments is shown in Figure 1. The scan function includes an ionization period $(\sim 10 \mathrm{~ms})$, a parent ion isolation period $(\sim 10 \mathrm{~ms})$, a collisional cooling period to allow parent ions to reach a stable internal energy distribution determined by the bath gas pressure, bath gas temperature and the trapping field $(\sim 10 \mathrm{~ms})$ [22], a collisional activation period, and finally, the analytical radiofrequency ramp that ejects the ions of increasing mass-to-charge ratio to the detector. Resonance excitation amplitude and duration are variables in the collisional activation step, Most of the data acquired for this study were obtained by using "key-sequence" programs that allow experimental parameters such as resonance excitation amplitude and resonance excitation duration to be incremented systematically during an experiment. These programs are available upon request to the authors.

\section{Threshold Resonance Excitation Amplitude Measurement}

The following discussion outlines how measurements of dissociation rate constants at different resonance

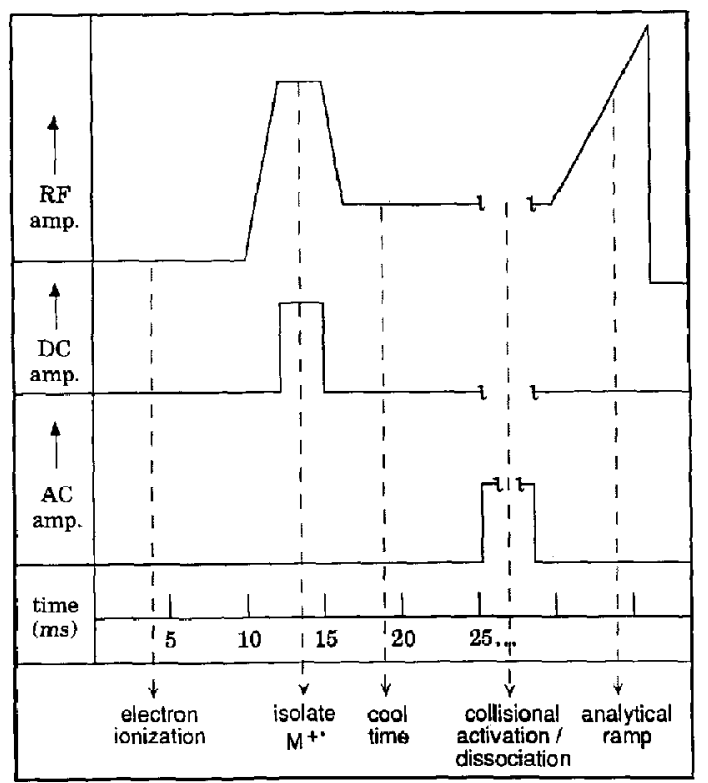

Figure 1. A generalized depiction of the sequence of events, generally referred to as the scan function, used in this study to measure decomposition kinetics. The radiofrequency amplitude refers to the $1.1 \mathrm{MHz}$ sine wave applied to the ring electrode, the direct current amplitude refers to a fixed potential added to the radiofrequency signal applied to the ring electrode, and the alternate current amplitude refers to the resonance excitation signal applied to the end-caps. excitation amplitudes are obtained and used to estimate a threshold resonance excitation amplitude. The description is broken into the major components of the measurement procedure.

Frequency optimization at various amplitudes. A requirement of critical importance in determining dissociation rate constants and threshold resonance excitation amplitudes using single frequency resonance excitation in the ion trap is to be "on-resonance." The excitation frequency must therefore be tuned to maximize power absorption by the parent ions of interest. Excitation frequency tuning is accomplished experimentally in the ITMS by varying the "delta frequency" in the ITMS scan table where the resonance excitation signal is applied to the endcaps. Dissociation rate constants determined with an "off-resonance" resonance excitation amplitude will be lower than the "on-resonance" rate constants and this would, in turn, lead to threshold resonance excitation amplitudes that are higher than the true value.

Typically, dissociation rate constants were determined at seven different resonance excitation amplitudes with the interval between resonance excitation amplitudes being $20 \mathrm{mV}$. We have noted, however, that the optimum resnnance excitation frequency varies nonlinearly with resonance excitation amplitude $(\sim 200$ $\mathrm{Hz}$ over a $180 \mathrm{mV}$ range) [18]. This trend was observed for all of the systems studied in this work. Thus, the resonance excitation frequency must be optimized for each resonance excitation amplitude used for a dissociation rate constant determination. An optimum resonance excitation frequency is selected by monitoring parent ion abundance while varying the resonance excitation frequency and determining the frequency where maximum loss of parent ion is observed. Initially, the optimum resonance excitation frequency can be found by varying the delta frequency in $100 \mathrm{~Hz}$ increments. The resonance excitation duration is then set to a value corresponding to $50 \%$ dissociation of the parent ion. An optimum resonance excitation frequency to the nearest $25 \mathrm{~Hz}$ is obtained by acquiring tandem mass spectra of the parent ions under "key-sequence" control where the delta frequency is scanned over a $400 \mathrm{~Hz}$ range in $25 \mathrm{~Hz}$ increments. The optimum resonance excitation frequencies determined in this step are then used to determine dissociation ratc constants, as described in the next section. It should also be noted that some shift in optimal resonance excitation frequency was observed as a function of time for resonance excitation amplitudes close to the threshold resonance excitation amplitude for the dissociation of styrene (i.e., $\sim 350 \mathrm{~Hz}$ over a $25 \mathrm{~ms}$ range of resonance excitation pulse duration). This variation was not observed to any appreciable extent in the other model dissociations. This observation may be related to the frequency shift observed with increasing resonance excitation amplitude. If there is a relationship between time and oscillatory amplitude, as there 


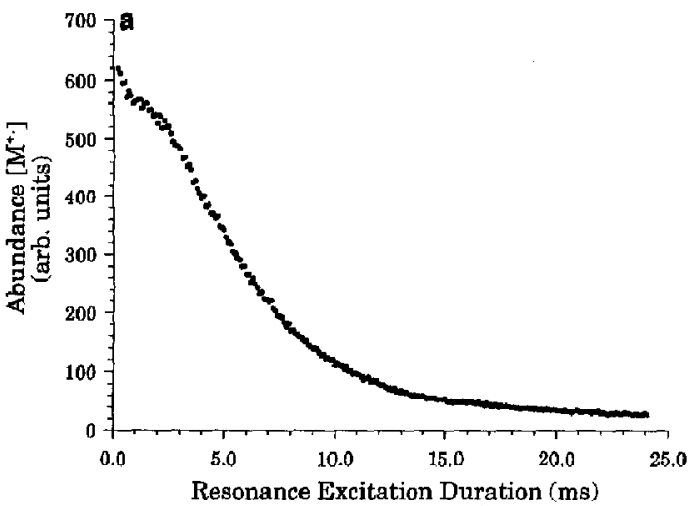

Figure 2. (a) A plot of the abundance of the n-butylbenzene molecular ion as a function of time using a resonance excitation amplitude of $150 \mathrm{mV}$. (b) A plot of the natural logarithm of the ratio of the n-butylbenzene molecular ion at time $t$ to that at time zero versus collisional activation time. (c) The same plot as shown in Figure $2 b$ showing the linear portion of the plot and a linear regression fit of the data.

must be as the resonance excitation signal is initiated, the optimum frequency would be expected to shift with time. The fact that no significant time dependence was observed for most of the systems described here may be related to the oscillatory amplitude required for dissociation. Ionized styrene has the highest critical energy of all of the systems studied and is therefore expected to require the highest oscillatory amplitude to induce dissociation. For this reason it is the most likely system to show a time dependence for optimum frequency. Experimentally, this problem was overcome by ramping the delta frequency (i.e., the optimal resonance excitation frequency) under key-sequence control while acquiring the dissociation rate constant data.

Dissociation rate constant determination at several resonance excitation amplitudes. It has been noted that a plot of abundance of parent ion versus resonance excitation pulse duration resembles an exponential decay [15]. An example of this type of plot for n-butylbenzene is provided in Figure 2a. A dissociation rate can be calculated by plotting the logarithm of the normalized parent ion abundance versus time (i.e., $\ln \left[\mathrm{P}_{t} / \mathrm{P}_{0}\right]$ versus the time in the scan function table that controls the resonance excitation pulse), as shown in Figure $2 b$. There is usually nonlinearity at very short resonance excitation times (i.e., $<2 \mathrm{~ms}$ ). This nonlinearity is due to an induction time where the parent ion population is being collisionally heated to the dissociation threshold energy [15]. Nonlinearity may also be observed at long times where most of the parent ion population has been dissociated. There is usually very good linearity between these extremes, as shown in Figure $2 c$. The slope of this line reflects the rate of the dissociation in units of reciprocal seconds. The helium buffer gas number density calculated from the ion gauge pressure reading is then used to calculate a phe-

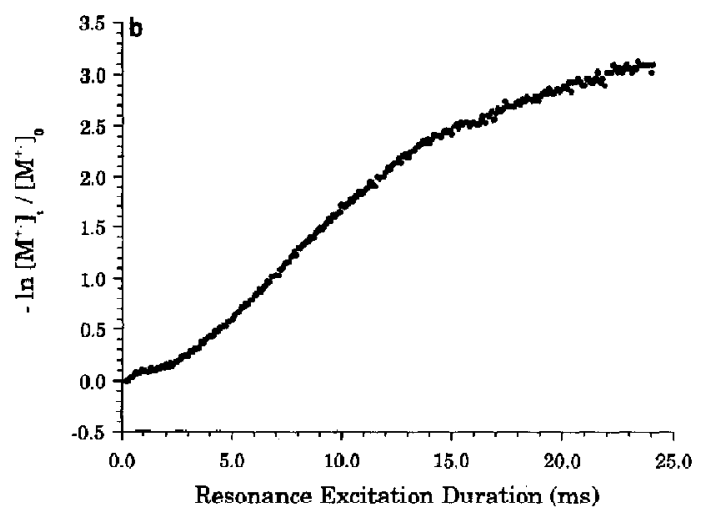

Figure 2. Continued.

nomenological rate constant for each resonance excitation amplitude in units of centimeters cubed per molecule per second. For example, the phenomenological dissociation rate constant determined for $n$-butylbenzene at $150 \mathrm{mV}$ as shown in Figure $2 \mathrm{c}$ is $6.61 \times$ $10^{-12} \mathrm{~cm}^{3} \mathrm{~mol}^{-1} \mathrm{~s}^{-1}$.

Plot $k_{o}$ versus resonance excitation amplitude and extrapolate to $k_{p}=0$. Once phenomenological rate constants $\left(k_{v}\right)$ have been determined for several resonance excitation amplitudes, a plot of $\mathbf{k}_{v}$ versus resonance excitation amplitude is prepared. The plot obtained for nitromethane over a broader range of resonance excitation amplitudes than normally acquired in this study is shown in Figure 3a. The $k_{v}$ versus $V$ plot is usually characterized, depending on the resonance excitation amplitudes selected, by a linear portion at intermediate resonance excitation amplitudes (e.g., Figure $3 \mathrm{~b}$ ) and a nonlinear portion at lower resonance excitation amplitudes (e.g., Figure 3c). At high resonance excitation amplitudes, the $\mathrm{k}_{\mathrm{v}}$ versus $\mathrm{V}$ and the $\ln \left[\mathrm{M}_{\mathrm{t}}^{+\cdot} / \mathrm{M}_{0}^{+\cdot}\right]$ versus $t$ become nonlinear again (e.g., Figure $3 a, 0.23$ to $0.29 \mathrm{~V}$ ).

The parameter of interest in this work is the "threshold resonance excitation amplitude" obtained

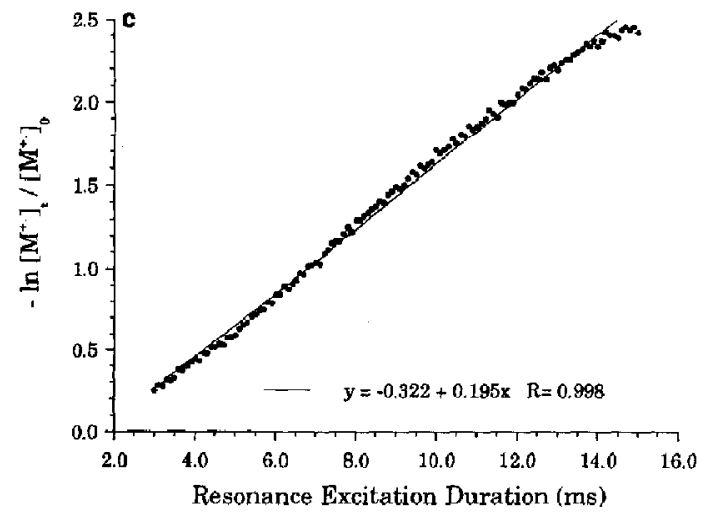

Figure 2, Continued. 


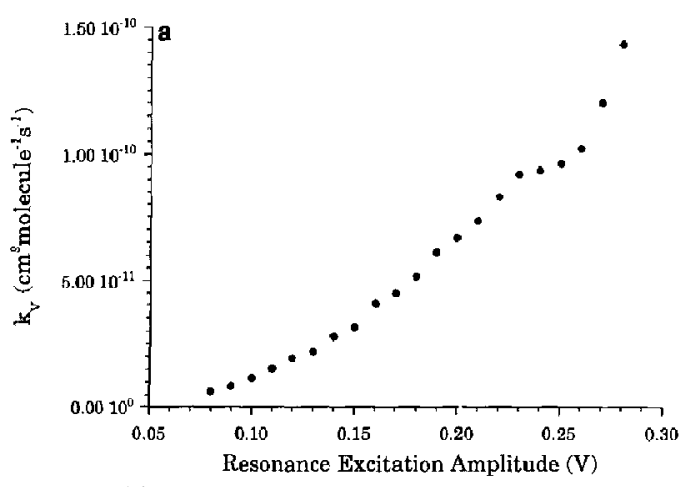

Figure 3. (a) A plot of the measured rate constant for decomposition of the nitromethane molecular ion as a function of resonance excitation amplitude. (b) The same plot as in Figure 3a showing the linear portion only along with a linear regression fit of the data. (c) The same plot as in Figure 3a showing the nonlinear portion at low resonance excitation amplitudes only and an extrapolation of the data to $k_{v}=0$ based on the curve fit shown.

by extrapolating the curve for the $\mathrm{k}_{\mathrm{v}}$ versus $\mathrm{V}$ plot to $k_{v}$ equal to zero (i.e., no dissociation). Because the $k_{v}$ versus $V$ curve is usually nonlinear near the threshold resonance excitation amplitude, most of the phenomenological rate constants should be determined at resonance excitation amplitudes near the threshold to define the curve. Threshold resonance excitation amplitudes were obtained in this study by use of a gaussian curve fit of the form:

$$
y=p_{1}+p_{2}\left(\exp \left[\left(x-p_{3}\right)^{2} / p_{4}^{2}\right]\right)
$$

where $p_{1}, p_{2}, p_{3}$, and $p_{4}$ are adjustable parameters and $\left(p_{1}+p_{2}\right)=0$ (so if $x=p_{3}, y=0$ ). For the nitromethane model dissociation, a linear extrapolation is clearly not indicated because there are data points below the threshold resonance excitation amplitude predicted from the linear curve fit shown in Figure $3 b$ (i.e., $0.107 \mathrm{~V}$ ). The threshold resonance excitation am-

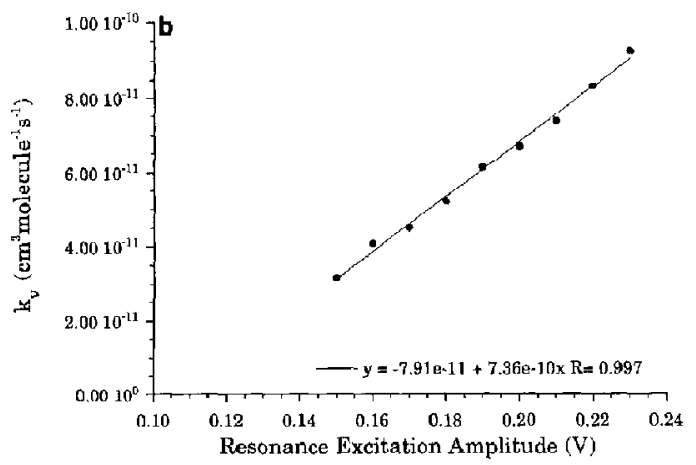

Figure 3. Continued. plitude from the nonlinear curve fit yields $0.053 \mathrm{~V}$, as shown in Figure 3c.

\section{Results and Discussion}

\section{Kesonance Excitation Frequency Shifts}

Optimization of the resonance excitation frequency is critically important in MS/MS experiments conducted in an ion trap mass spectrometer [23-25]. Resonance excitation is accomplished in the ion trap by calculating the theoretical secular frequency for the parent ion and applying an alternating current (ac) voltage with that frequency to the endcap electrodes. The ac frequency is then tuncd to obtain maximum absorption by the parent ion (i.e, maximum dissociation of the parent ion).

The need for tuning of the resonance excitation frequency has slowed the development of on-line separations combined with MS/MS using the ion trap mass spectrometer, although methods for rapid optimization have been reported [26, 27]. MS/MS using broad-band excitation has also been reported as a convenient method for resonance excitation that does not require tuning of individual frequencies [28, 29]. However, for the experiments reported here, tuning time is not a critical parameter. The principal concern in these experiments is that the resonance excitation frequency be tuned at each resonance excitation amplitude. The resonance excitation absorption curves [18] clearly demonstrate that the optimal resonance excitation frequency changes as a function of resonance excitation amplitude while all other instrumental parameters are held constant. Some variation in the optimal resonance excitation frequency with excitation time was also observed for the styrente system, as shown in Figure 4. This behavior was not observed to any appreciable extent in the other systems investigated in this study. One of the differentiating factors is that the dissociation of styrene has the largest critical energy for dissociation of the systems studied.

The resonance excitation peak shape for parent ions stored at $q_{2}$ values of less than 0.4 is expected to be lorentzian for an ion trap with a pure quadrupole field

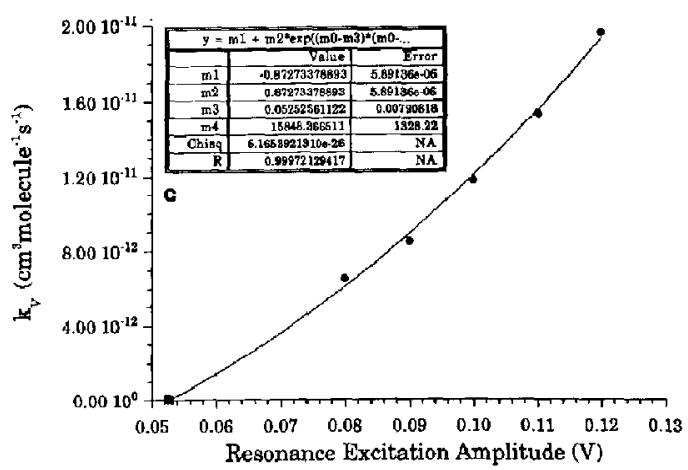

Figure 3. Continued. 


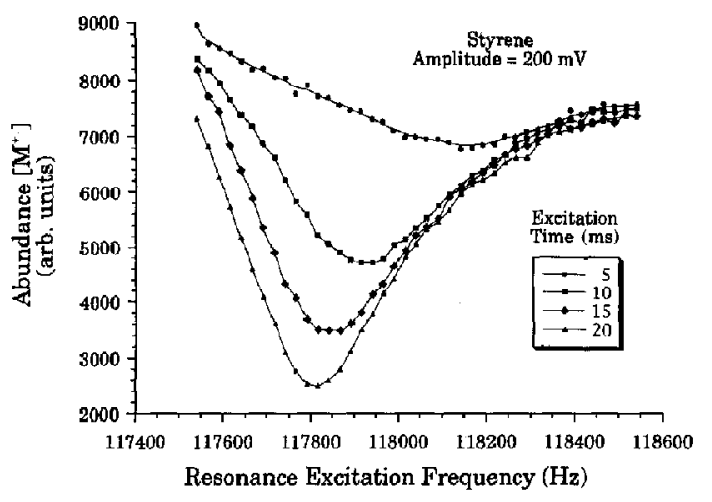

Figure 4. A series of plibts of the abundance of the molecular ion of styrene as a function of frequency at a resonance excitation amplitude of $200 \mathrm{mV}$. Fach curve represents a different resonance excitation period.

and is expected to be independent of amplitude [30]. The peak shapes shown in Figure 4 were generated from spectra acquired at a fixed excitation amplitude but different excitation times. These peaks are clearly not symmetrical nor are their maxima centered at the same frequency. We have reported elsewhere a similar observation from experiments conducted with fixed excitation times and variable excitation amplitudes [18]. A detailed rationale for the shapes of these peaks and their dependence upon amplitude is not the intent of this article. However, the instrument used in this work employs an ion trap with end-caps intentionally spaced farther apart than necessary for a pure quadrupole field [31]. This creates a weak contribution from an octopole field, which is known to cause a frequency shift that is correlated with excitation amplitude [32]. The observed variation in resonance excitation profiles observed here is therefore at least qualitatively accounted for by the presence of higher order multipole fields. For the purpose of making quantitative measurements of relative dissociation energies, the dependence of frequency on amplitude is a complication. Future studies will therefore be directed toward making measurements with an ion trap with electrode-spacings intended to minimize contributions from higher order multipole fields.

\section{Nonlinearity at High Resonance Excitation Amplitudes}

The plot of dissociation rate constants versus resonance excitation amplitude for the nitromethane system shown in Figure 3a consists of three distinct parts. The rate constants plotted from 0.08 to $0.12 \mathrm{~V}$ approach the threshold resonance excitation amplitude in a nonlinear fashion, as shown in Figure 3b. A linear relationship between dissociation rate constants and resonance excitation amplitude is then observed from 0.14 to 0.22 V. However, substantial nonlinearity at resonance exci- tation amplitudes greater than $0.22 \mathrm{~V}$ is evident in the plot shown in Figure 3a. Several factors can come into play in determining the shape of the curve at high resonance excitation amplitudes. As mentioned above, the ion absorption profile shifts and broadens as amplitude increases in the Finnigan geometry ion trap and could therefore alter the characteristics of the ion cloud in resonance. The significance of this behavior on the energy distribution of the ion cloud is unclear at this point. Furthermore, the probability for ion ejection becomes more significant. In any case, the focus of the present study is on the behavior of the ions near threshold. The dissociation rate constants reported here were calculated using the disappearance of the parent ion and thus assume that resonance ejection is not occurring. For species with relatively low to moderate critical energies in which the loss of parent ion can be accounted for by appearance of product ions, this assumption would appear to be valid.

\section{Effects of Experimental Parameters}

The threshold resonance excitation amplitudes reported here provide a basis of comparison of dissociation energetics that are independent of the amplitude and duration of the resonance excitation signal. Other experimental factors that affect collisional dissociation in the ion trap are helium buffer gas pressure, the temperature of the helium, and the secular frequency of the ion as determined by the Mathieu q stability parameter. The pressure and temperature of the helium buffer gas were held constant in this study. These parameters may affect the internal energy of the stored parent ions prior to collisional activation and thus can affect the value of the measured threshold resonance excitation amplitudes. The effects of these parameters on threshold resonance excitation amplitude are the subject of further study.

The effect of changing the $q_{x}$ value of the parent ions during the dissociation step has been studied for the n-butylbenzene system. Table 1 shows the linear regression results obtained from the $k_{v}$ versus $V$ plots

Table 1. Linear regression results obtained for plots of $k_{\mathrm{v}}$ versus $V$ using data acquired at several $q$ values ${ }^{a}$

\begin{tabular}{lccc}
\hline $\mathrm{q}$ & $\mathrm{x}_{\text {int }}(\mathrm{V})$ & $\mathrm{Y}_{\text {int }}\left(\mathrm{cm}^{3} \mathrm{~mol}^{-1} \mathrm{~s}^{-1}\right)$ & $\mathrm{k}^{\left(\mathrm{cm}^{3} \mathrm{~mol}^{-1} \mathrm{~s}^{-1} \mathrm{~V}^{-1}\right)}$ \\
\hline \hline 0.20329 & 0.1038 & $-1.364 \times 10^{-11}$ & $1.314 \times 10^{-10}$ \\
0.25073 & 0.1010 & $-1.784 \times 10^{-11}$ & $1.766 \times 10^{-10}$ \\
0.30087 & 0.1020 & $-2.166 \times 10^{-11}$ & $2.124 \times 10^{-10}$ \\
0.35034 & 0.1071 & $-2.953 \times 10^{-11}$ & $2.758 \times 10^{-10}$ \\
0.40049 & 0.1127 & $-3.737 \times 10^{-11}$ & $3.316 \times 10^{-10}$ \\
$\begin{array}{l}\text { Average } \\
\text { Standard }\end{array}$ & 0.1053 & $-2.400 \times 10^{-11}$ & $2.255 \times 10^{-10}$ \\
deviation & 0.0047 & $0.949 \times 10^{-11}$ & $0.793 \times 10^{-10}$ \\
$\begin{array}{l}\text { \% Relative } \\
\text { standard }\end{array}$ & & & \\
deviation & 4.5 & 39.5 & 35.2 \\
\hline
\end{tabular}

${ }^{a}$ The system being studied was the dissociation of n-butylbenzene molecular ion. 
for $n$-butylbenzene at five different $g_{z}$ values. The $k_{v}$ versus $V$ plots for n-butylbenzene did not show appreciable nonlinearity near the threshold, as was the case for the other systems, so a linear regression fit was acceptable. Only a $4.5 \%$ relative standard deviation was observed for these determinations, which indicates that the threshold resonance excitation amplitudes are insensitive to changes in the $q_{z}$ of the parent ion. The slope and $y$ intercept of the lines, however, change with the $q_{z}$ of the parent ion. The slopes of the lines obtained in the $\mathbf{k}_{v}$ versus $V$ plots for the $n$ butylbenzene system, in units of centimeters cubed per molecule per second per volt, are plotted versus $q_{3}$ in Figure 5. So while the parent ion population continues to dissociate at the same threshold resonance excitation amplitude, the rate at which the parent ions dissociate increases with increasing $\mathrm{q}_{z}$.

\section{Empirical Correlation of Threshold Resonance Excitation Amplitude with Literature Critical Energies}

An empirical correlation of the threshold resonance excitation amplitudes obtained in the ion trap for several model dissociations was made with literature critical dissociation energies. The model dissociations selected for this work yield predominantly one product ion and have fairly well-characterized energetics. A list of model dissociations, literature critical energies, and threshold resonance excitation amplitudes measured by using the method outlined in this article is provided in Table 2 [33-40]. The model dissociations span a range of critical energies of approximately $2 \mathrm{eV}$. The first model dissociation listed in Table 2 is loss of - $\mathrm{C}_{2} \mathrm{H}_{5}$ from ionized tetraethylsilane with a critical energy of $0.5 \mathrm{eV}$. Slow dissociation of this ion is observed with no resonance excitation applied under the trapping conditions used in this study. Clearly, a fraction of the internal energy distribution of this ion in

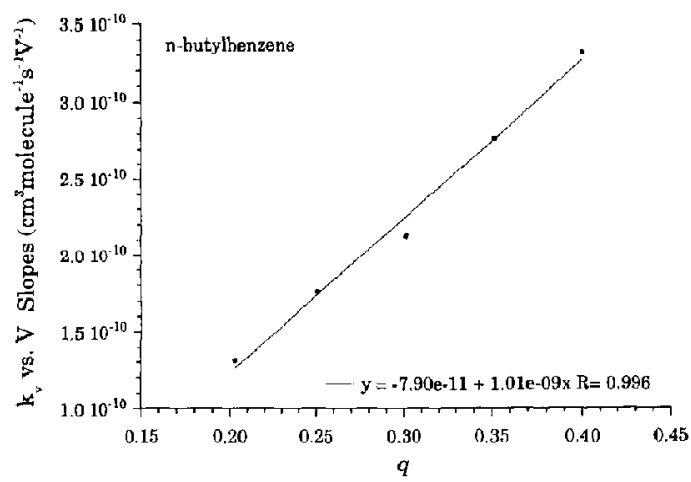

Figure 5. A plot of the slopes derived from the $k_{v}$ versus $V$ plots at various values of $\mathrm{q}_{2}$ for the n-butylbenzene molecular ion.
Table 2. Literature critical energy and threshold resonance excitation amplitudes for several model dissociations ${ }^{a}$

\begin{tabular}{|c|c|c|}
\hline $\begin{array}{l}\text { Compound name } \\
\text { (dissociation) }\end{array}$ & $\begin{array}{l}E_{0} \text { lit. } \\
\text { (eV) }\end{array}$ & $\begin{array}{l}\text { TREA } \\
\text { (mV) }\end{array}$ \\
\hline $\begin{array}{l}\text { Tetraethylsilane } \\
\text { (loss of } \cdot \mathrm{C}_{2} \mathrm{H}_{5} \text { ) }\end{array}$ & $0.5[33]$ & $0^{b}$ \\
\hline $\begin{array}{l}\text { Nitromethane } \\
\text { (loss of } \cdot \mathrm{CH}_{3} \mathrm{O} \text { ) }\end{array}$ & $0.71 \pm 0.03[34]$ & $52.5 \pm 7.9$ \\
\hline $\begin{array}{l}\text { Allyl chloride } \\
\text { (loss of } \cdot \mathrm{Cl} \text { ) }\end{array}$ & $1.01 \pm 0.05[35]$ & $88.40 \pm 2.9$ \\
\hline $\begin{array}{l}\text { n-Butylbenzene } \\
\text { (loss of } \mathrm{C}_{3} \mathrm{H}_{6} \text { ) }\end{array}$ & $1.10 \pm 0.10[36]$ & $105.3 \pm 9.4$ \\
\hline $\begin{array}{l}\text { Ethylbenzene } \\
\left(\text { loss of } \cdot \mathrm{CH}_{3}\right)\end{array}$ & $1.30 \pm 0.02[37]$ & $137.5 \pm 8.7$ \\
\hline $\begin{array}{l}\text { Toluene } \\
\text { (loss of } \cdot H \text { ) }\end{array}$ & $1.70 \pm 0.06[38]$ & $159.7 \pm 6.4$ \\
\hline $\begin{array}{l}\text { Styrene } \\
\text { (loss of } \mathrm{C}_{2} \mathrm{H}_{2} \text { ) }\end{array}$ & $\begin{array}{c}2.43 \pm 0.05[39] \\
(2.7 \pm 0.6)[40]\end{array}$ & $180.6 \pm 10.2$ \\
\hline
\end{tabular}

a The molecular ion formed by electron ionization was the parent ion in all cases.

${ }^{b}$ Tetraethylsilane dissociates spontaneously under the conditions used in these experiments. This value should be considered an upper bound.

$E_{0}$ lit = Literature critical energy with reported error estimate

TREA = Threshold resonance excitation amplitude with estimated error from curve fit statistics.

the absence of collisional activation extends beyond the dissociation energy. Indeed, calculations of internal energy distributions as a function of temperature for ions with the number of degrees of freedom comparable to tetraethylsilane indicate that a temperature of $350 \mathrm{~K}$, for example, gives an average energy less than $0.5 \mathrm{eV}$, but the high energy tail of the distribution

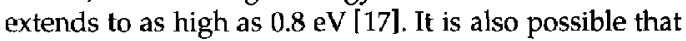
some dissociation of the tetraethylsilane parent ion may occur due to activation of the parent ion during the isolation step. In the case of tetraethylsilane, an extrapolation to a negative threshold amplitude would probably give a good fit to the curve correlating literature critical energies with experimentally derived threshold amplitudes (see below). At this point in our work, however, we felt it was important to be consistent in our method for determining the threshold voltage for those ions which show clear nonlinear behavior near threshold, and the gaussian curve fit does not allow for a negative number. Furthermore, it is unclear at this stage of our understanding how well dissociation energies for ions that dissociate in the absence of collisional activation can be estimated from rates derived by using collisional activation. We have therefore arbitrarily assigned this ion a threshold resonance excitation amplitude of $0 \mathrm{~V}$ and regard this situation as a special case meriting further attention.

A plot of literature critical energies versus the threshold resonance excitation amplitudes is provided in Figure 6. Error bars were established for the literature values plotted in this figure by using the reported error estimates. The error bars for the resonance excita- 


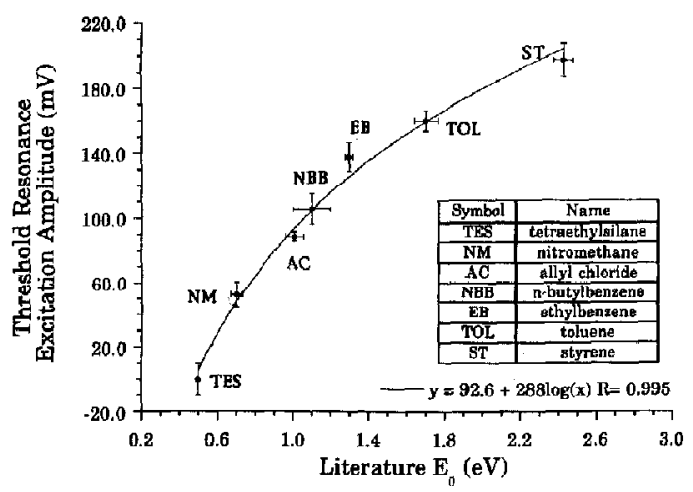

Figure 6. A plot of measured threshold resonance excitation amplitudes versus literature critical energy values for decomposition of the molecular ions of the indicated compounds.

tion amplitudes were estimated from the curve fit statistics. No error bars were plotted for the tetraethylsilane system because no error estimate was reported for the literature value. There is a good logarithmic correlation between these parameters. This curve reflects the deposition of internal energy with increasing resonance excitation amplitude in the ion trap mass spectrometer. Model systems composed of large organic ions with dissociation energies higher than 2.5 $\mathrm{eV}$ were avoided because the critical energies reported in the literature in this range are more likely to contain significant kinetic shift and because the likelihood for error in establishing a threshold critical amplitude is higher due to possible contributions from ion ejection. The ion trap dissociation energies are likely to be less subject to kinetic shift due to the slow collisional activation mechanism in the ion trap.

A good example of a system with significant kinetic shift is the loss of $\mathrm{H}$ from 1-methylnaphthalene. Huang and Dunbar [41] recently reported a kinetic shift of $2.15 \mathrm{eV}$ for this system relative to a conventional threshold measurement of $4.4 \mathrm{eV}$ that was reported in the literature. The value cited by Huang and Dunbar was $2.25 \mathrm{eV}$ and was obtained by time-resolved photodissociation in an ion cyclotron resonance (ICR) spectrometer at two different wavelengths accompanied by RRKM modeling. The dissociation energy for this process is anticipated to be similar to the analogous reaction with ionized toluene which has a critical energy of $1.7 \mathrm{eV}$. The threshold resonance excitation amplitude obtained for methylnaphthalene in this study was $155.4 \pm 12.2 \mathrm{mV}$. The threshold resonance excitation amplitude obtained for toluene, as shown in Table 1, was $159.7 \pm 6.4 \mathrm{mV}$. If the curve plotted in Figure 6 is used to correlate threshold resonance excitation amplitude with dissociation energy, the result would be $1.69 \pm 0.17 \mathrm{eV}$. Thus, the ion trap collisional activation results appear to support the results of Huang and Dunbar, at least qualitatively, that there is a large kinetic shift associated with the conventional critical energy measurements for 1-methylnaphthalene $[42,43]$.

\section{Relationship Between Threshold Resonance Excitation Amplitude and Critical Energy}

A qualitative relationship between an ion's kinetic stability and the resonance excitation amplitude necessary for efficient dissociation in the ion trap is widely recognized. An a priori quantitative relationship is not yet in hand. However, the experimental results given here suggest that at least a semiquantitative relationship might be obtained. Before that is possible, however, we must understand the physical meaning of the threshold resonance excitation amplitude (i.e., how it might be related either directly or indirectly to the critical energy for dissociation of an ion). We outline here our current working hypothesis and speculate on why there might be a logarithmic correlation between threshold resonance excitation amplitude and critical energy, as has been observed empirically.

Two hypothetical $P(\epsilon)$ versus $\epsilon$ curves, both Boltzmann internal energy distributions, are shown in Figure 7 and represent parent ion populations before resonance excitation and during collisional activation once a steady-state condition is reached. (It has not yet been demonstrated that ion internal energy distributions are Boltzmann and can be described by a temperature. However, it seems likely that a population of polyatomic ions will assume a Boltzmann distribution under conditions in which both multiple heating and cooling collisions occur.) The curve indicating lower internal energies represents the distribution of internal energy for parent ions stored within the trap prior to resonance excitation. The higher distribution depicted in Figure 7 represents the distribution of internal ener-

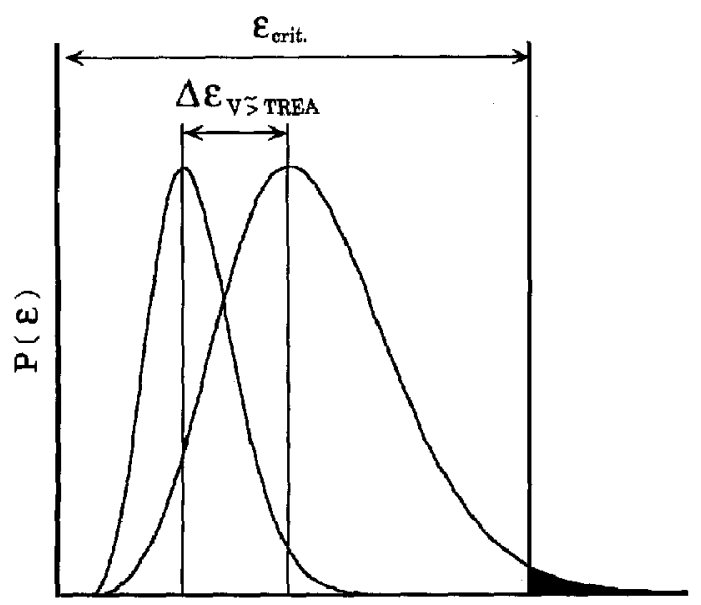

$\varepsilon$

Figure 7. A plot of two Boltzmann distributions indicating the qualitative relationship between the measured threshold resonance excitation energy and the critical energy for decomposition. The threshold resonance excitation energy is a measure of the change in temperature required to achieve a measurable rate of decomposition for the molecular ion. TREA $=$ threshold resonance excitation amplitude. 
gies for parent ions undergoing resonance excitation once the distribution has achieved a steady state. There is, of course, the period in which the distribution is transformed from the low energy distribution to the high energy distribution. However, in the absence of a dissociation reaction, there is no mechanism preventing the final distribution from being achieved provided the irradiation time is sufficient. Our interpretation of the threshold resonance excitation measurement is that it reflects a parent ion "temperature" at which a negligible number of parent ions have sufficient internal energy to fragment but one in which a slight increase in temperature would cause dissociation. The $k_{v}$ values observed reflect the rate at which parent ions achieve sufficient energy to fragment.

Figure 7 also indicates a hypothetical critical energy for dissociation which is measured relative to the zero point energy of the ion. The amplitude of the resonance excitation shown in Figure 7 is slightly in excess of the threshold resonance excitation amplitude because some of the parent ions possess internal energies in excess of the critical energy for dissociation (as shown by the highlighted region). We interpret the threshold resonance excitation amplitude as a measurement of the change in the internal energy distributions from the initial stored parent ion internal energy distribution to the distribution just before dissociation is observed. If these internal energy distributions can be characterized by temperatures, the threshold resonance excitation amplitude is a measure of $\Delta T$ and clearly not a direct measurement of the critical energy. Provided all parent ions start at the same temperature, however, there is a correlation between $\Delta \mathrm{T}$ and critical energy because they are proportional. This would suggest that the measured threshold resonance excitation amplitude should be dependent upon the initial parent ion temperature. Anticipating that this would be the case, we purposely performed these experiments under conditions that would minimize differences in parent ion temperature. Systematic variations in parent ion temperature to test the foregoing argument is the subject of future study.

The shape of the curve in Figure 6, which compares threshold resonance excitation amplitude values with literature critical energies, is of particular interest from the point of view of understanding collisional activation in the ion trap. If the ordinate and abscissa of Figure 6 were switched, it would be apparent that critical energies are reached with a greater than linear dependence on resonance excitation amplitude. If the interpretation described above is correct, the threshold resonance excitation amplitude value represents a change in ion temperature. Both the average internal energy and the high energy tail of the distribution for a polyatomic system increase in a nonlinear fashion with temperature bearing striking resemblance to the shape of Figure 6 with reversed ordinate and abscissa. The relationship between resonance excitation amplitude and temperature (in the absence of fragmentation) is therefore highly relevant. We are currently performing modeling studies and experiments to test some of the reasoning offered here to explain our results and to explore the possibility for deriving a quantitative relationship between ion temperature and resonance excitation amplitude under typical ion trap conditions.

\section{Conclusions}

The experimental results reported here indicate that there is a quantitative relationship between threshold resonance excitation energies and the kinetic stability of polyatomic ions. The ability to order, and perhaps even quantify, ion stability via careful measurements of threshold resonance excitation amplitude values enhances the utility of the quadrupole ion trap in gasphase ion chemistry research. However, a dependence of the optimal resonance excitation frequency on amplitude, presumably due to the presence of higher order multipole fields in commercially available ion traps, creates experimental complications in the measurements.

The types of information that can be obtained both from the threshold resonance excitation amplitude values and the amplitude-dependent rates of dissociation remain to be fully delineated. However, this approach may prove to be a useful method for characterizing fundamental properties of gaseous ions whereby, like Huang and Dunbar's slow multiphoton experiment in the ICR, the excitation process is slow relative to dissociation. For this reason, information derived from ion trap data may complement results obtained from experiments in which the excitation step is fast relative to dissociation. Furthermore, study in this area both from experimental and theoretical points of view promises to provide new insights into the excitation and deexcitation processes in the ion trap under collisional activation conditions.

\section{Acknowledgments}

The authors acknowledge helpful discussions with Dr. Douglas E. Goeringer. Research was sponsored by the U.S. Department of Energy, Office of Basic Energy Sciences, under Contract DEAC05-84OR21400 with Martin Marietta Energy Systems.

\section{References}

1. Lias, S. G.; Bartmess, J. E.; Liebman, J. F.; Holmes, J. L.; Levin, R. D.; Mallard, W. G. J. Phys. Chem. Ref. Data 1988, 17, Suppl. $1,5$.

2. Rosenstock, H. M. Int. J. Mass Spectrom. Ion Phys. 1976, 20, 139.

3. Gentry, W. R. In Gas Phase Ion Chemistry, Vol. 2; Bowers, M. T., Ed. Academic Press: New York, 1979; p 221.

4. Futrell, J. H. In Gaseous Ion Chemistry and Mass Spectrometry; Futrell, J. H., Ed. John Wiley: New York, 1986; p 155.

5. Graul, S. T.; Squires, R. R. Int. J. Mass Spectrom. Ion Proc. 1987, $81,183$.

6. Chupka, W. A. J. Chem. Phys. 1959, 30, 191. 
7. Lifshitz, C.; Long, F. A. J. Chem. Phys. 1964, 41, 2468.

8. Chupka, W. A. J. Chem. Phys. 1971, 54, 1936.

9. Dunbar, R. C. J. Chem. Phys. 1991, 95, 2537.

10. Dunbar, R. C.; Zaniewski, R. C. J. Chem. Phys. 1992, 96, 5069.

11. Gauthier, J. W.; Trautman, T. R.; Jacobson, D. B. Anal. Chim. Acta 1991, 246, 211.

12. Thorne, L. R.; Beauchamp, J. L. In Gas Phase Ion Chemistry, Vol. 3; Bowers, M. T., Ed. Academic Press: New York, 1984; p 41.

13. McLuckey, S. A.; Glish, G. L.; Van Berkel, G. J. Int. I. Mass Spectrom. Ion Proc. 1991, 106, 213.

14. Gronowska, J.; Paradisi, C.; Traldi, P.; Vettori, U. Rapid Commun. Mass Spectrom. 1990, 4, 306.

15. Franklin, A. M.; Todd, J. F. J.; March, R. E. Proceedings of the 41st Annual ASMS Conference on Mass Spectrometry and Allied Topics; San Francisco, May 30-June 4, 1993; p 925.

16. March, R. E. Int. J. Mass Spectrom. Ion Proc. 1992, $118 / 119$, 71.

17. Goeringer, D, E.; McLuckey, S. A. Unpublished results; Oak Ridge National Laboratory, 1993.

18. Williams, J. D.; Cox, K. A.; Cooks, R. G.; McLuckey, S. A.; Hart, K. J.; Goeringer, D. E. Anal. Chem., in press.

19. Louris, J. N.; Cooks, R. G.; Syka, J. E. P.; Kelley, P. E.; Stafford, G. C., Jr.; Todd, J. F. J. Anal. Chem. 1987, 59, 1677.

20. Bartmess, J. E.; Georgiadis, R. M. Vacuum 1983, 33, 149.

21. March, R E.; Hughes, R. J. Quadrupole Ion Storage Mass Spectrometry; John Wiley: New York, 1989.

22. McLuckey, S. A.; Asano, K. G.; Glish, G. L.; Bartmess, J. E. Int. J. Mass Spectrom. Ion Proc. 1991, 109, 171.

23. Todd. J. F. J. Mass Spectrom. Ren. 1991, 70, 3.

24. March, R. E. Int. J. Mass Spectrom. Ion Proc. 1992, 118 / 119, 71.

25. Johnson, J. V.; Yost, R. A.; Kelley, P. E.; Bradford, D. C. Anal. Chem. 1990, 62, 2162.

26. Yates, N. A.; Yost, R. A.; Bradshaw, S. C.; Tucker, D. B.
Proceedings of the 39th Annual ASMS Conference on Mass Spectrometry and Allied Topics; Nashville, TN, May 19-24, 1991; p 132.

27. Penman, A. Di Todd, J. F. J.; Thorner, D. A.; Smith, R. D. Rapid Commun. Mass Spectrom. 1990, 4, 415.

28. McLuckey, S. A.; Goeringer, D. E.; Glish, G. L. Anal. Chem. 1992, 64, 1455.

29. Van Berkel, G. J.; Goeringer, D. E. Anal. Chim. Acta 1993, 277. 41.

30. Goeringer, D. E.; Whitten, W. B.; Ramsey, J. M.; McLuckey, S. A.; Glish, G. L. Anal. Chem. 1992, 64, 1434.

31. Louris, J. N.; Stafford, G. C.; Syka, J. E. P.; Taylor, D. Proceedings of the 40th Annual ASMS Conference on Mass Spectrometry and Allied Topics; Washington, DC, May 31, 1992; p 1003.

32. Franzen, J. Int. J. Mass Spectrom. Ion Proc. 1991, 106, 93.

33. de Ridder, J. J.; Dijkstra, G. Recl. Trav. Chim. 1967, 86, 737.

34. Nicholson, A. J. C. Recent Developments in Mass Spectrometry; Ogata, K, Hayakawa, T., Eds. University Park Press: Baltimore, 1970; p 745.

35. Buff, R. D.; Parr, A. C.; Jason, A. J. Int. J. Mass Spectrom. Ion Proc. 1981, 40, 31.

36. Chen, J. H.; Hayes, J. D.; Dunbar, R. C. J. Phys. Chem. 1984, 88,4759 .

37. McLoughlin, R. G.; Morrison; J. D.; Traeger, J. C. Org. Mass Spectrom. 1979, 14, 104.

38. Bombach, R; Dannacher, J.; Stadelmann, J. P. J. Am. Chem. Soc. 1983, 105, 4205.

39. Dunbar, R. C. J. Phys. Chem. 1990, 94, 3283.

40. Dunbar, R. C.; Zartewski, R. C. J. Chem. Phys. 1992, 96, 5069.

41. Huang, F. S.; Dunbar, R. C. J. Am. Chem. Soc. 1990, 112, 8167.

42. Koppel, C.; Schwarz, H.; Bohlmann, F. Org. Mass Spectrom. $1974,8,25$.

43. Loudon, A. G.; Mazengo, R. Z. Org. Mass Spectrom, 1974, 8, 179. 